\title{
Differential Effect of Hypoxia in Human and Mouse Vascular Smooth Muscle Cell Migration through LRP1-pPyk2-MMP-9 Axis
}

\author{
E. Revuelta-López, J. Castellano, D. de Gonzalo-Calvo, L. Nasarre, \\ L. Badimon, and V. Llorente-Cortés
}

Cardiovascular Research Center (CSIC-ICCC), IIB-Sant Pau, 08025 Barcelona, Spain

Correspondence should be addressed to V. Llorente-Cortés; cllorente@csic-iccc.org

Received 19 June 2014; Accepted 18 November 2014

Academic Editor: Antonio Lepedda

This Conference Paper is based on a presentation given by V. Llorente-Cortés at "LIAC Meeting on Vascular Research 2013" held from 18 September 2013 to 21 September 2013 in Alghero, Italy.

Copyright (C) 2015 E. Revuelta-López et al. This is an open access article distributed under the Creative Commons Attribution License, which permits unrestricted use, distribution, and reproduction in any medium, provided the original work is properly cited.

LRP1-pPyk2 axis is essential for the upregulatory effect of hypoxia on MMP-9 activation and human VSMC (hVSMC) migration. Currently, there are not efficient models for the translational study of atherosclerosis. The morphological and physiological features of atherosclerosis are different between human and animal models, particularly in mouse models. Therefore, the aim of current investigation was to compare the effect of hypoxia on LRP1-Pyk2-MMP-9 axis in human and mouse vascular smooth muscle cells (mVSMC) and its consequences on VSMC migration. We demonstrated that hypoxic modulation of LRP1-pPyk2-MMP-9 axis is opposite between hVSMC and mVSMC. The modulation of LRP1/pPyk2 levels by hypoxia is positive in hVSMC but negative in mVSMC. We showed that the inverse effect of LRP1/pPyk2 axis is associated with a differential effect of hypoxia on MMP-9 expression and activation. Hypoxia-induced MMP-9 activation was concomitant with an increased hVSMC migratory capacity. Surprisingly, mVSMC migrate under hypoxic conditions despite the downregulatory effect of hypoxia on MMP-9 expression or activation. Our results highlight the crucial role of LRP1-pPyk2-MMP-9 axis in vascular cell migration. In addition, we propose that the extrapolation of results from animal models to humans is not suitable for this specific mechanism in hypoxia-related vascular conditions.

\section{Introduction}

Atherosclerosis is included in a group of diseases that share pathophysiological mechanisms linked to the deregulation of vascular extracellular matrix remodeling in response to hypoxia [1]. Vascular smooth muscle cells (VSMC) are one of the main cellular determinants of arterial wall pathology. In particular, these cells play a crucial role in the modulation of extracellular matrix composition. VSMC modify extracellular matrix altering protein synthesis or degradation in response to atherogenic stimuli [2]. We have previously shown that human VSMC (hVSMC) internalize huge amounts of cholesterol esters from aggregated LDL through low density lipoprotein receptor-related protein 1 (LRP1) upregulation [3]. The induction of hVSMC-derived foam cells reduces the expression and activation of metalloproteinase9 (MMP-9) and, thus, hVSMC migration [4]. The arterial wall integrity is importantly affected by the balance between VSMC migration and proliferation. This balance is fundamental for intimal thickening.

VSMC proliferation, migration, and survival are tightly modulated by matrix-degrading metalloproteinases (MMPs). MMPs play a key role in vascular remodeling through selective cleavage of extracellular matrix components [5]. One key receptor in the modulation of MMP-9 and MMP-2 activity is LRP1 $[6,7]$. It has been demonstrated that LRP1 deficiency causes MMP-9 overexpression in mouse macrophages [8], inhibition of cell migration in rat Schwan cells [9], and, by downregulation of MMP-2 expression, decreased angiotensin II-induced migration of rat aortic smooth muscle cells [10]. 
Our group has previously demonstrated that LRP1 is upregulated by hypoxia through hypoxia-inducible factor(HIF-) $1 \alpha$ in hVSMC [11]. By increasing intracellular calcium levels through LRP1 [12], hypoxia alters proline-rich tyrosine kinase 2 (Pyk2) phosphorylation [13]. Pyk2 has been involved in several physiological processes, including cell growth, differentiation, metabolism, and cytoskeleton function. Previous results from our group have demonstrated that LRP1pPyk2 axis is essential for the upregulatory effect of hypoxia on MMP-9 activation and hVSMC migration [14].

Nowadays, there is not gold standard animal model for atherosclerosis investigation. The morphological and pathologic features of the atherosclerotic process differ between human and laboratory animals [15]. Mouse models have a very thin and VSMC-poor intima in early stages of atherosclerosis while VSMC are the major component in the intima of human atherosclerosis-prone arteries [16]. In addition, contrary to humans, proliferation of VSMC and production of extracellular matrix occur after the accumulation of foamy macrophages [15]. Due to the relevance of animal models in atherosclerosis studies, the investigation of the different pathological mechanisms of the atherosclerotic process in humans and animals models is essential.

The aim of this study was to compare the effect of hypoxia on LRP1-Pyk2-MMP-9 axis in hVSMC and mouse VSMC (mVSMC) and its consequences on VSMC migration.

\section{Material and Methods}

2.1. Culture of Human Vascular Smooth Muscle Cells. The media layer of atherosclerotic plaque-free human arteries was processed using a modification of the explant method for isolation of VSMC as previously described [17, 18]. Vessels were obtained at surgery from patients undergoing cardiac transplantation at Hospital de la Santa Creu i Sant Pau, Barcelona, and cut into $1 \mathrm{~mm} \times 1 \mathrm{~mm}$ tissue pieces in the culture unit. These explants of medial tissue were incubated at $37^{\circ} \mathrm{C}$ in a humidified atmosphere of $5 \% \mathrm{CO}_{2}$. After 1 week, cells started to grow out radially from the explants and proliferated covering the culture dishes. Cells used in the experiments were between the fourth passage and sixth passage and were characterized by Western blot analysis for specific differentiation markers ( $\alpha$-actin, $45 \mathrm{kDa}$, and calponin, $33 \mathrm{kDa}$ ). Cell monolayers were grown to $80 \%$ confluence in medium 199 supplemented with 20\% (v/v) FBS, $2 \%$ (v/v) human serum, $2 \mathrm{mmol} / \mathrm{L}$ L-glutamine, $100 \mathrm{U} / \mathrm{mL}$ penicillin $\mathrm{G}$, and $100 \mu \mathrm{g} / \mathrm{mL}$ streptomycin. The protocol was approved by the Research Ethics Committee at the study center and was conducted in accordance with the Declaration of Helsinki.

\subsection{Culture of Mouse Vascular Smooth Muscle Cells. Animal} management was in accordance with institutional guidelines and regulations, and animal procedures were approved by the Research Ethics Committee. mVSMC were harvested from the aorta of wild type C57BL/6 mice as previously described [19]. mVSMC were cultured in medium DMEM supplemented with $10 \%$ (v/v) FBS, 2 mmol/L L-glutamine,
$100 \mathrm{U} / \mathrm{mL}$ penicillin $\mathrm{G}$, and $100 \mu \mathrm{g} / \mathrm{mL}$ streptomycin. Cells used in the experiments were between the fifth passage and sixth passage, and cell monolayers were grown to $80 \%$ confluence.

2.3. Cell Exposure to Normoxic and Hypoxic Conditions. hVSMC or mVSMC were deprived of serum once they reached $80 \%$ confluence. Cells were exposed to normoxia $\left(21 \% \mathrm{O}_{2}\right)$ in an incubator with gas mixtures consisting of $74 \%$ $\mathrm{N}_{2}$ and $5 \% \mathrm{CO}_{2}$ or to hypoxia $\left(1 \% \mathrm{O}_{2}\right)$ in a hypoxic/anoxic workstation: H35 (Don Whitley Scientific Ltd.) with 94\% $\mathrm{N}_{2}$ and $5 \% \mathrm{CO}_{2}$. Cells were then harvested by scraping in TriPure Isolation Reagent (Roche Molecular Diagnostics) for PCR and Western blot. Culture supernatants were finally collected for zymographic studies. In some experiments, quiescent VSMC were untreated or treated with PP2 (Calbiochem) or U0126 (Cell Signaling).

2.4. Western Blotting. After normoxic or hypoxic treatment, cells were washed with PBS and total protein was isolated by TriPure Isolation Reagent (Roche Molecular Biochemicals) according to the manufacturer's instructions. Equivalent amounts of total protein $(25 \mu \mathrm{g})$ were electrophoresed on polyacrylamide gels for SDS-PAGE. The samples were electrotransferred to nitrocellulose, and the membranes were saturated at room temperature for 1 hour in TBS-T $(20 \mathrm{mM}$

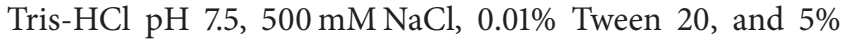
nonfat milk). Western blot analyses were performed with specific monoclonal antibodies (Table 1) and the corresponding secondary antibodies (1:10000 dilution; Dako). Equal protein loading was verified with Ponceau staining and by performing blotting of Western blots with $\beta$-tubulin (Abcam, ab6046, 1:1000 dilution). Bands were detected using the ECL prime Western blotting detection reagent (Amersham) and quantified by densitometry using ChemiDoc system and Quantity-One software (Bio-Rad). Results are expressed as arbitrary units of intensity.

2.5. Real Time PCR. Serum-deprived cells under normoxia or hypoxia were washed with PBS and total RNA was isolated by TriPure Isolation Reagent (Roche Molecular Biochemicals) according to the manufacturer's instructions. Reverse transcription was performed using the high capacity cDNA reverse transcription kit (Applied Biosystems), and the reaction mix was subjected to quantitative real time PCR to detect LRP1, MMP-9, and MMP-2 mRNA expression using the assays on demand (Applied Biosystems) detailed in Table 2. Human TATA-binding protein TBP expression (4326322E) and ARBP expression (Rn00821065_g1) (Applied Biosystems) were unaltered by hypoxia and used as internal control for human and mice gene amplification, respectively. Real time PCR was performed using $1 \mu \mathrm{L} /$ well of reverse transcription products in $10 \mu \mathrm{L}$ of TaqMan PCR master mix (PE Biosystem) with primers at $300 \mathrm{nM}$ and probe at $200 \mathrm{nM}$. PCR was performed in a PCR-7600HT sequence detection system (Abiprism, Applied Biosystems) at $95^{\circ} \mathrm{C}$ for 10 minutes for AmpliTaq Gold activation and then run for 40 cycles at $95^{\circ} \mathrm{C}$ for 15 seconds and $60^{\circ} \mathrm{C}$ for 1 minute. The relative levels 
TABLE 1: Primary antibodies used in Western blot analysis.

\begin{tabular}{lcc}
\hline Primary antibody & Company & Reference \\
\hline LRP1 $\beta$-chain & Epitomics & $\# 2703-1$ \\
Phospho-Pyk2 & Cell Signalling Technology & $\# 3291$ \\
Total Pyk2 & Cell Signalling Technology & $\# 3480$ \\
Phospho-ERK1,2 & Cell Signalling Technology & $\# 9101$ \\
Total ERK1,2 & Assay Designs & KAP-MA001 \\
$\beta$-tubulin & Abcam & Ab6046 \\
LRP1 $\beta$-chain & Fitzgerald & 10R-L107c \\
\hline
\end{tabular}

TABLE 2: References of assays on demand used in real time PCR.

\begin{tabular}{lcc}
\hline Assay on demand & Species & Reference \\
\hline MMP-9 & Human & Hs00234579_m1 \\
MMP-2 & Human & Hs00234422_m1 \\
SREBP-1 & Human & Hs00231674_m1 \\
SREBP-2 & Human & Hs00190237_ml \\
MMP-9 & Mouse & Mm00600163_m1 \\
MMP-2 & Mouse & Mm00439498_m1 \\
SREBP-1 & Mouse & Mm01138344_m1 \\
SREBP-2 & Mouse & Mm00439498_m1 \\
TBP & Human & 4326322E \\
ARBP & Mouse & Rn00821065_g1 \\
\hline
\end{tabular}

of gene expression were quantified and analyzed using SDS 2.4 software. The real time value for each sample was averaged and compared using the $\mathrm{C}_{\mathrm{T}}$ method, where the amount of target RNA $\left(2^{-\Delta \Delta C T}\right)$ was normalized to the endogenous control $\left(\Delta \mathrm{C}_{\mathrm{T}}\right)$ and related to the amount of target gene in the cells.

2.6. Gelatin Zymography. The relative activity of MMP-9 and MMP-2 in the cell culture supernatant was measured by zymography. Media derived from each condition were dialyzed against $0.01 \mathrm{M} \mathrm{NH}_{4} \mathrm{HCO}_{3}$, lyophilized, and resuspended in RIPA buffer $(150 \mathrm{mM} \mathrm{NaCl}, 1 \%(\mathrm{v} / \mathrm{v})$ Triton X$100,0.5 \%(\mathrm{w} / \mathrm{v})$ sodium deoxycholate, $0.1 \%$ (w/v) SDS, $2 \mathrm{mM}$ EDTA, and $50 \mathrm{mM}$ Tris-HCl pH 8) supplemented with complete protease inhibitor cocktail (Roche). Supernatants were mixed with $2 \mathrm{X}$ nonreducing loading buffer $(10 \%(\mathrm{w} / \mathrm{v})$ SDS, $0.5 \mathrm{M}$ Tris- $\mathrm{HCl} \mathrm{pH} \mathrm{6.8,0.5 \%} \mathrm{(w/v)} \mathrm{bromophenol} \mathrm{blue,} \mathrm{and}$ $10 \%(\mathrm{w} / \mathrm{v})$ Sucrose) and equal volumes of each sample were loaded in $10 \%(\mathrm{v} / \mathrm{v})$ SDS-polyacrylamide gel with $1 \mathrm{mg} / \mathrm{mL}$ porcine skin type A gelatin (Sigma-Aldrich) as a substrate for MMPs enzymatic activity and run at $4^{\circ} \mathrm{C}$ for $4-6 \mathrm{~h}$. After electrophoresis, the gels were rinsed twice for $30 \mathrm{~min}$ at room temperature in 2.5\% Triton X-100 (Sigma) and then incubated in substrate buffer $\left(50 \mathrm{mM}\right.$ Tris- $\mathrm{HCl}, 10 \mathrm{mM} \mathrm{CaCl}_{2}$ (Merk), and $0.02 \%(w / v) \mathrm{N}_{3} \mathrm{Na}$ (Fluka), $\mathrm{pH}$ 8) for $18-20 \mathrm{~h}$ at $37^{\circ} \mathrm{C}$. They were dyed with $10 \%$ acetic acid with one tablet of PhastGel Blue R (GE Healthcare). Areas of gelatinolytic activity appeared as clear bands on a blue background where the protease has digested the substrate. Gels were finally scanned with a GS-800 calibrated imaging densitometer (Bio-Rad) and quantitative densitometric analysis of digested bands was performed using Quantity-One software (BioRad).

2.7. Cell Migration and Wound Repair Assays. VSMC were seeded on glass-bottom sterile culture dishes. Confluent cells were scratched to create a double-sided wound [4] and washed with PBS. hVSMC and mVSMC were grown in M199 and DMEM, respectively, supplemented with FBS (10\%), penicillin/streptomycin (1\%), and L-glutamine (1\%). Photographs were taken after indicated times, and cell migration and wound repair were analyzed measuring the injured area covered by cells counted from the wounding borders with the Image J software.

2.8. Statistical Analysis. Results are expressed as mean \pm SEM. The number of experiments is shown in every case. Statistical differences between control and treated groups were analyzed by the nonparametric Mann-Whitney $U$ test for paired data. A $P$ value less than 0.05 was considered significant.

\section{Results}

3.1. Effect of Hypoxia in Intracellular Signaling in hVSMC and mVSMC. Western blot analysis showed that hypoxia increased Pyk2 phosphorylation from 4 to 32 hours in hVSMC (Figures 1(a) and 1(c)). This effect was concomitant with LRP1 upregulation (Figures 1(a) and 1(b)). Conversely, phosphorylated Pyk2 (Figures 1(e) and 1(g)) and LRP1 (Figures 1(e) and 1(f)) protein levels were significantly decreased at 16 and 32 hours in mVSMC exposed to hypoxia.

The ratio phosphorylated/total ERK1,2 was significantly upregulated by hypoxia at 8 hours in hVSMC (Figures 1(a) and $1(\mathrm{~d})$ ). In contrast, the increase of phosphorylation was maintained until 16 hours in mVSMC (Figures $1(\mathrm{e})$ and $1(\mathrm{~h})$ ).

3.2. Effect of Hypoxia on HIF-1 $\alpha$ and Sterol Regulatory Element Binding Proteins 1 and 2 (SREBP-1 and SREBP-2) in hVSMC and $m V S M C$. Hypoxia strongly induced HIF- $1 \alpha$ protein levels between 3 and 8 hours in both hVSMC (Figure 2(a)) and mVSMC (Figure 2(b)). Concerning SREBPs, hypoxia did not exert any significant effect on SREBP-1 mRNA levels either in hVSMC (Figure 2(c)) or in mVSMC (Figure 2(d)). In contrast, hypoxia significantly reduced SREBP-2 mRNA levels in both hVSMC (Figure 2(e)) and mVSMC (Figure 2(f)), although later in hVSMC.

3.3. Effect of Hypoxia on MMP Expression and Activity in hVSMC and mVSMC. Quantitative RT-PCR analysis showed that hypoxia specifically upregulated MMP-9 mRNA (Figure 3(a)) but not MMP-2 mRNA level in hVSMC (Figure 3(a)). Accordingly, zymography analysis showed an increase in MMP-9 but not in MMP-2 activity in hypoxic hVSMC (Figures 3(b) and 3(c)). In contrast to the upregulatory effect of hypoxia on both MMP-9 expression and activity in hVSMC, MMP-9 and MMP-2 mRNA expression (Figure 3(d)) and MMP-9/MMP-2 activities (Figures 3(e) and 3(f)) were downregulated in hypoxic mVSMC. 
Human VSMC

Time (hours)

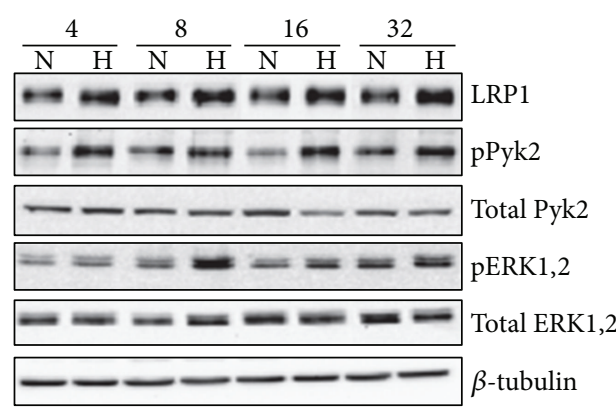

(a)

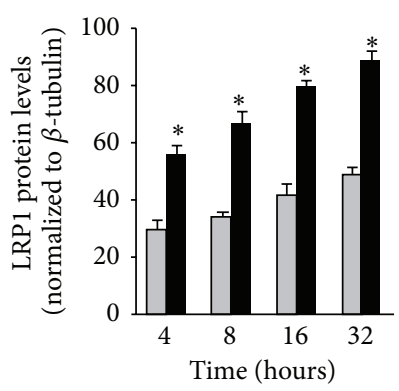

- N: normoxia H: hypoxia

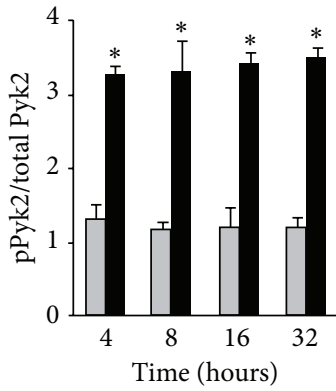

- N: normoxia

口 H: hypoxia

(c)

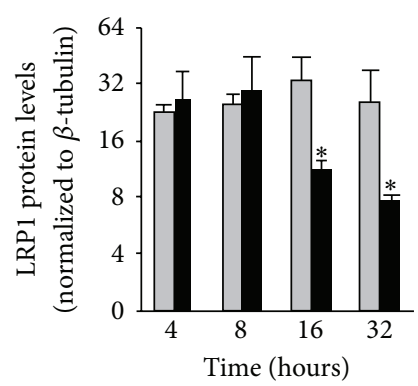

- N: normoxia 口 H: hypoxia
Mouse VSMC

Time (hours)

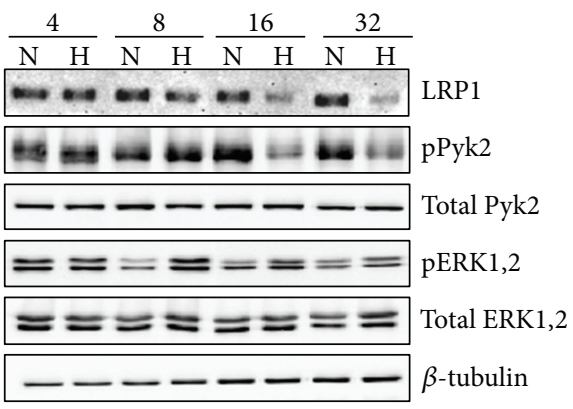

(e) (b)

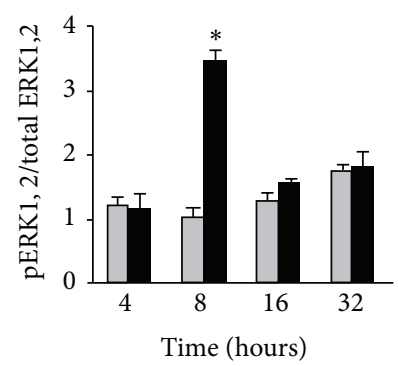

- N: normoxia

$\square \mathrm{H}$ : hypoxia

(d)

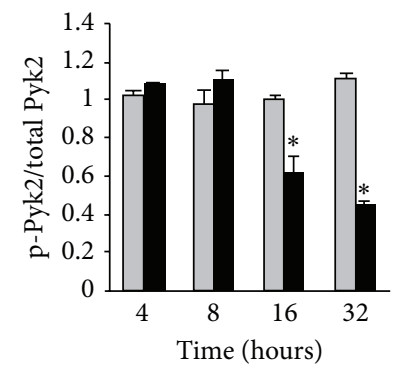

- N: normoxia

口 H: hypoxia

(g)

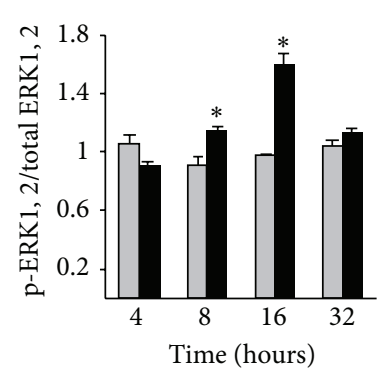

- N: normoxia

$\square \mathrm{H}$ : hypoxia

(h)

FIgURE 1: Time-course of hypoxia induced Pyk2 and ERK1,2 phosphorylation in hVSMC and mVSMC. hVSMC and mVSMC were exposed to either normoxia or hypoxia for 4, 8, 16, and 32 hours. ((a), (e)) Representative Western blot analysis showing LRP1, pPyk2, total Pyk2, pERK1,2, and total ERK1,2 bands. $\beta$-tubulin was detected as loading control. Bar graphs show quantification of LRP1 ((b), (f)), pPyk2/total Pyk2 ratio ((c), (g)), and pERK1,2/total ERK1,2 ((d), (h)) in normoxic (gray bars) and hypoxic (black bars) conditions. Results are expressed as mean \pm SEM of two independent experiments performed in triplicate. N, normoxia; $\mathrm{H}$, hypoxia and ${ }^{*} P<0.05$ versus normoxic cells.

3.4. Impact of PP2 and UO126 Treatment on Hypoxia Induced $h$ VSMC and $m$ VSMC Migration. Wound repair experiments performed using an in vitro scrape-injury model showed that hVSMC exposed to hypoxia for $18 \mathrm{~h}$ had a higher percentage of recovered area than normoxic cells (Figures 4(a) and 4(b)). However, mVSMC exposed to hypoxia for 3, 6, and 9 hours had a greater migratory capacity than normoxic mVSMC. However, the difference did not reach statistical significance (Figures 4(c) and 4(d)).
Exposure to PP2 completely and specifically prevented hypoxia-induced Pyk2 phosphorylation (data not shown). PP2 strongly impaired migration of both normoxic and hypoxic hVSMC (Figures 4(a) and 4(b)).

UO126, a selective inhibitor of ERK1 and ERK2 phosphorylation, blocked ERK1,2 phosphorylation in hVSMC (data not shown). UO126 decreased hVSMC migration in both normoxic and hypoxic conditions (Figures 4(a) and 4(b)). In $\mathrm{mVSMC}$, the percentage of recovered area in hypoxic cells 


\section{Human VSMC}
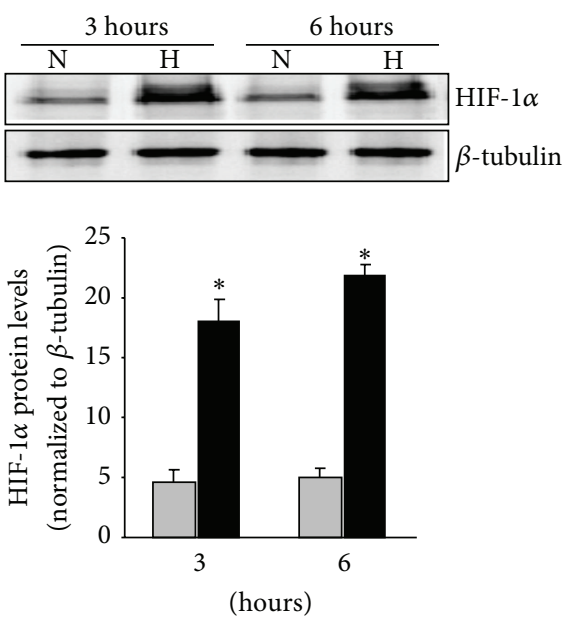

$\square \mathrm{N}$ : normoxia

- H: hypoxia

(a)

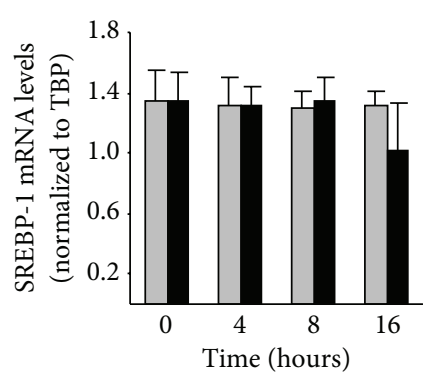

$\square$ N: normoxia

- H: hypoxia

(c)

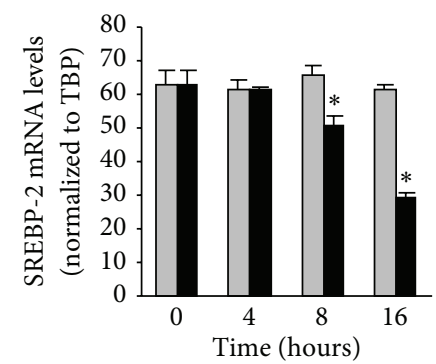

$\square \mathrm{N}$ : normoxia

- H: hypoxia

(e)

\section{Mouse VSMC}
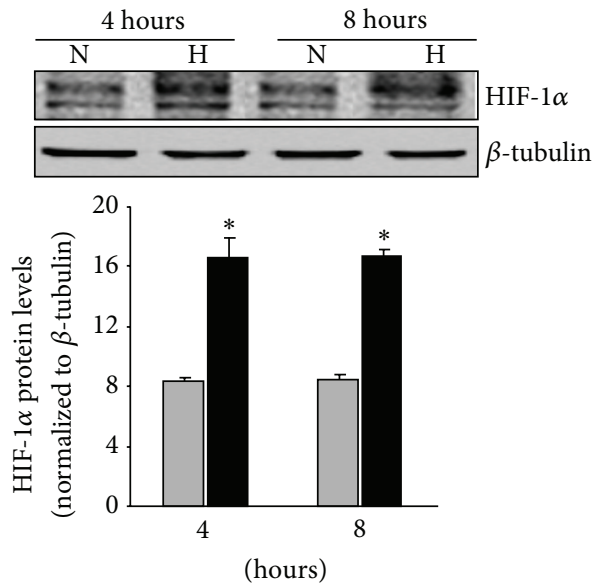

$\square \mathrm{N}$ : normoxia

- H: hypoxia

(b)

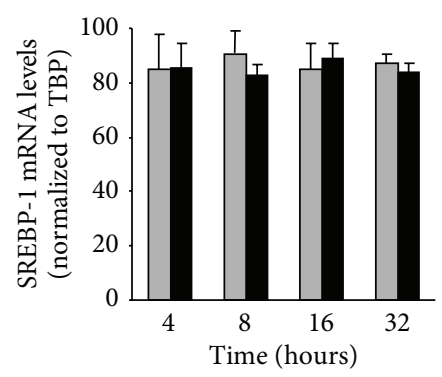

$\square \mathrm{N}$ : normoxia

- H: hypoxia

(d)

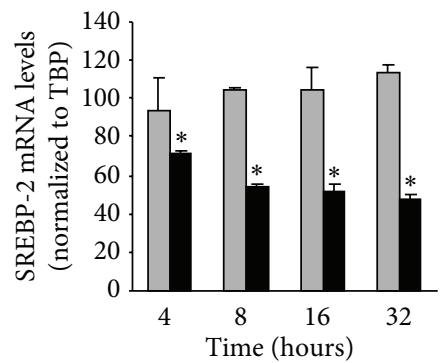

$\square$ N: normoxia

H: hypoxia

(f)

FIGURE 2: Time-course of hypoxic modulation of HIF- $1 \alpha$ and SREBPs levels in hVSMC and mVSMC. hVSMC and mVSMC were exposed to either normoxia or hypoxia for increasing times. ((a), (b)) Representative Western blot analysis showing HIF-1 $\alpha$ levels. $\beta$-tubulin was used as loading control. Bar graphs represent quantification of HIF- $1 \alpha$ levels in normoxia (N, gray bars) or hypoxia (H, black bars). Real time PCR experiments showing the effect of hypoxia on SREBP-1 ((c), (d)) and SREBP-2 ((e), (f)) mRNA levels in hVSMC ((c) and (e), resp.) and $\operatorname{mVSMC}\left((\mathrm{d})\right.$ and $(\mathrm{f})$, resp.). Results from two independent experiments performed in duplicate are expressed as mean \pm SEM. ${ }^{*} P<0.05$ versus normoxic cells. 
Human VSMC

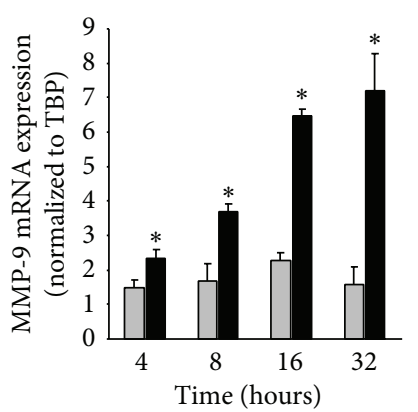

(a)

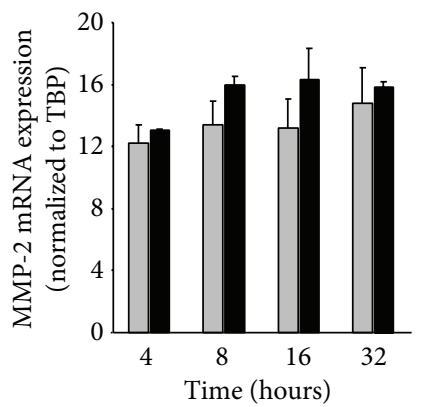

\begin{tabular}{|c|c|}
\hline \multicolumn{2}{|l|}{ Time (hours) } \\
\hline $8 \quad 16$ & \\
\hline 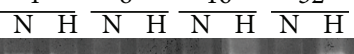 & \\
\hline$-m-m-5-6$ & $\begin{array}{l}\text { MMP-9 } \\
(105 \mathrm{kDa})\end{array}$ \\
\hline$-6=$ & \\
\hline
\end{tabular}

(b)

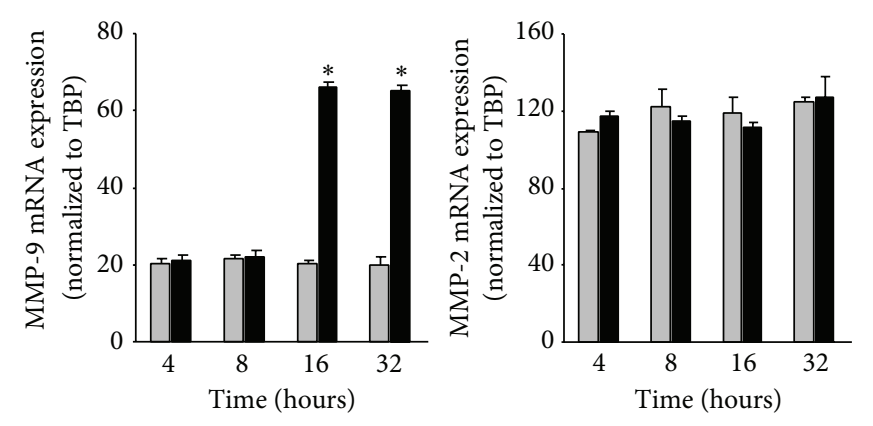

(c)

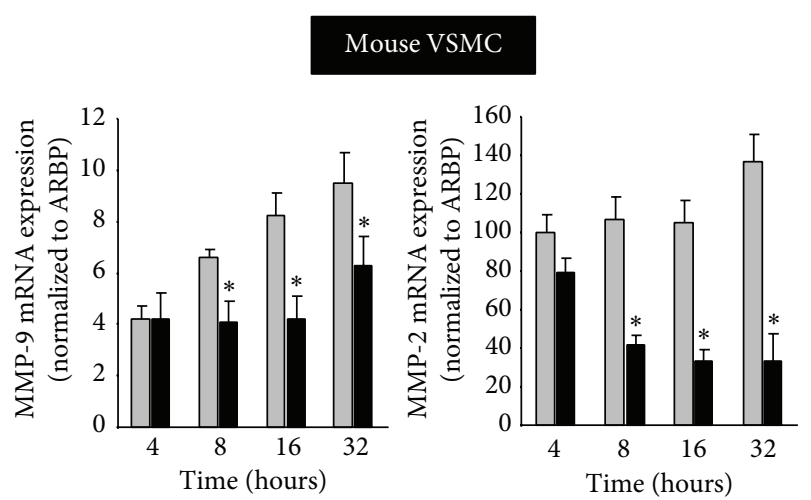

(d)

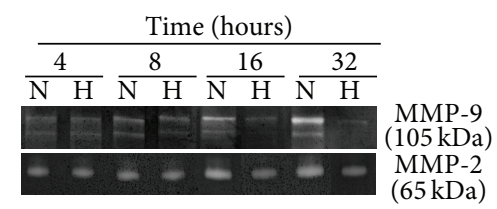

(e)
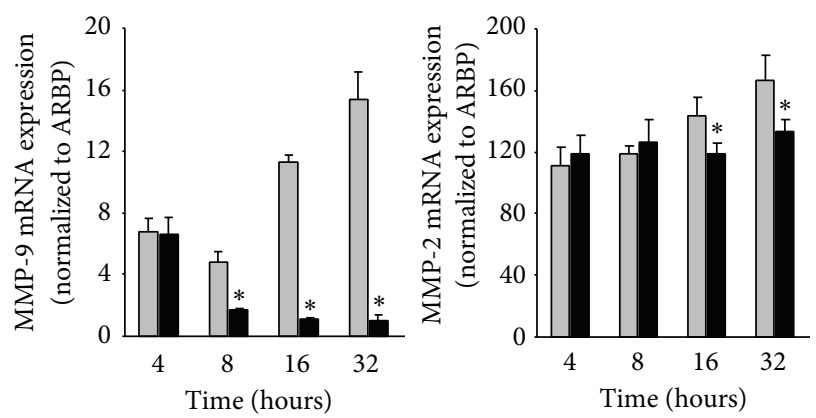

$(\mathrm{f})$

FIGURE 3: Time-course of the hypoxic modulation of MMP-9/MMP-2 expression and activation in hVSMC and mVSMC. hVSMC and mVSMC were exposed to either normoxia or hypoxia for increasing times and harvested to assess MMP-9 and MMP-2 expression using quantitative RT-PCR ((a) and (d), resp.). Endogenous gene reference was TBP and $A R B P$ for human and mouse, respectively. Representative zymographic analysis ((b) and (e), resp.) detecting MMP-9 and MMP-2 in conditioned media from normoxic and hypoxic cells and bar graphs showing the quantification of bands ((c) and (f), resp.). Results from two experiments performed in duplicate were expressed as mean \pm SEM. ${ }^{*} P<0.05$ versus normoxic cells.

was similar to that seen in normoxic mVSMC, and it was reduced in both normoxic and hypoxic cells with the UO126 treatment (Figures 4(c) and 4(d)).

\section{Discussion}

The main finding of current study is that the modulation of LRP1-pPyk2-MMP-9 axis by hypoxia is opposite in hVSMC and mVSMC. These opposite effects of hypoxia may repercute in a differential migratory response of hVSMC and mVSMC to hypoxia. These results have important conceptual implications. The extrapolation of data from animal models to humans is not suitable for this specific mechanism in hypoxia-related vascular diseases. Our results support previous investigations showing crucial differences between murine models and humans in vascular morphology [20], in the relative contribution of VSMC and macrophages to the development of atherosclerotic plaques at early stages [16], in foam macrophage formation and accumulation [15], and in the composition of the arterial intima [20].

Results from the present study show that the modulation of LRP1/pPyk2 levels by hypoxia is positive in hVSMC but negative in mVSMC. We have previously shown that HIF- $1 \alpha$ mediates hypoxia-induced LRP1 overexpression in hVSMC and HL-1 cells [11, 21]. HIF-1 $\alpha$ overexpression depends on intracellular calcium concentrations that rapidly and persistently increase in response to hypoxia [22,23]. An increase in calcium cytosolic levels also underlies Pyk2 phosphorylation [24]. It remains to be elucidated why Pyk2 phosphorylation is not increased in mVSMC despite HIF-1 $\alpha$ overaccumulating under hypoxic conditions.

Other transcription factors involved in the modulation of LRP1 transcription are SREBPs. Our group has reported that SREBPs negatively modulate LRP1 promoter transcription $[18,25]$. Results from the present study showed that hypoxia negatively downregulates SREBP-2 mRNA expression in 


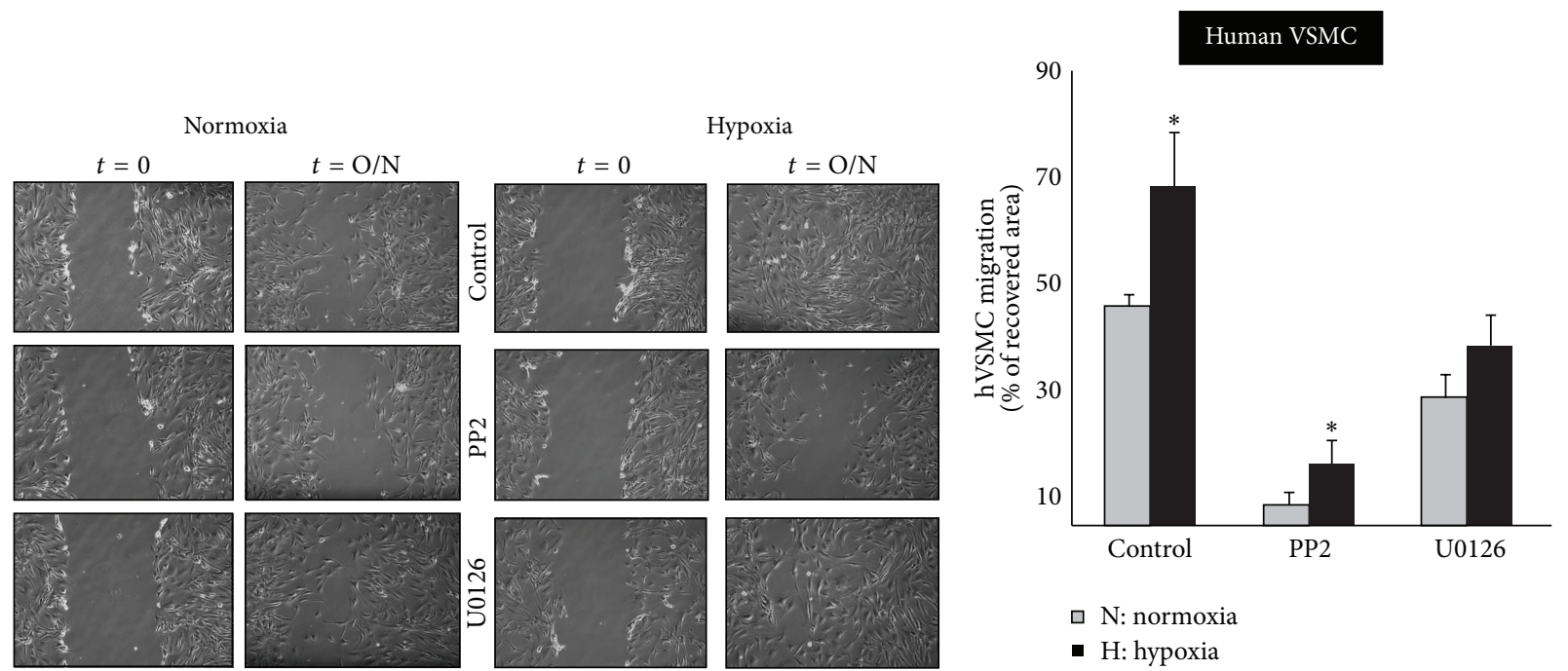

(a)

(b)

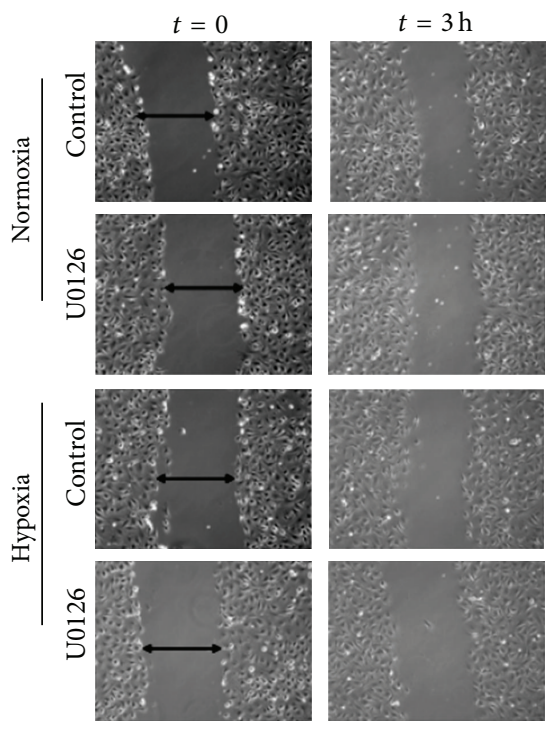

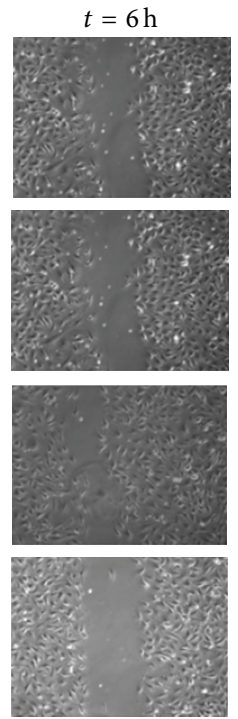

(c)

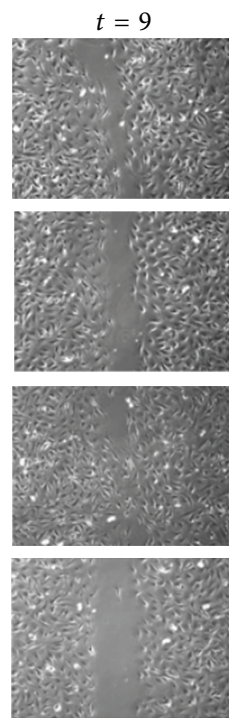

Mouse VSMC

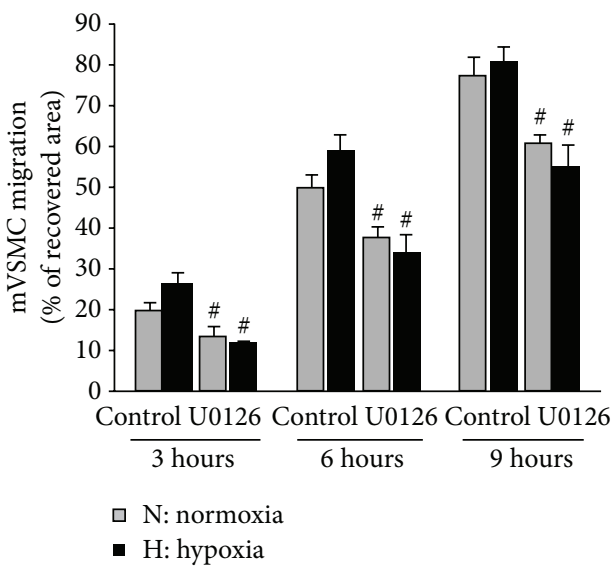

(d)

FIGURE 4: Effect of PP2 and U0126 on hypoxia-induced VSMC migration. Quiescent hVSMC or mVSMC were untreated or treated with PP2 $(50 \mu \mathrm{M})$ or U0126 $(10 \mu \mathrm{M})$ for different times under normoxic or hypoxic conditions. Phase-contrast microscopy images showing the migratory capacity of control, PP2-treated and U0126-treated hVSMC ((a) and (b)), and mVSMC ((c) and (d)) in normoxia and hypoxia and bar graphs show quantification of cell migration rate over time. Data from two independent experiments performed in triplicate are expressed as mean \pm SEM. ${ }^{*} P<0.05$ versus normoxic cells, ${ }^{\#} P<0.05$ versus control.

hVSMC and mVSMC. There are controversial results in the literature concerning the effect of hypoxia on SREBP expression. One of the variables that influences the hypoxic effect is cell-type [26,27]. Indeed, differences in the modulation of SREBPs by hypoxia explain why LRP1 is downregulated in cerebral VSMC [28] but upregulated in coronary VSMC [11]. According to the negative modulatory effect of SREBP-2 on LRP1 transcription, we would expect an upregulatory effect of hypoxia on LRP1 expression in both species. However, we observed an upregulatory effect of hypoxia on LRP1 upregulation in human but not in mVSMC. Since SRE sequences in mouse and human LRP1 promoter are homologous, the differential effect of hypoxia-induced SREBP decay between human and mouse VSMC could be explained by the effects of hypoxia on the levels and/or activity of other transcription factors that cooperate with SREBPs like Sp or KLF transcription factors. Further studies are required to explain these species-dependent divergent results.

Despite these differences, hypoxia-induced Pyk2 phosphorylation seems to be modulated by LRP1. The capacity of LRP1 to modulate Pyk2 phosphorylation may be explained by the capacity of LRP1 $\beta$-chain to retain calcium in its fifth and sixth CR-domains [29]. Therefore, it is expected that LRP1 upregulation in hVSMC will favor higher calcium 

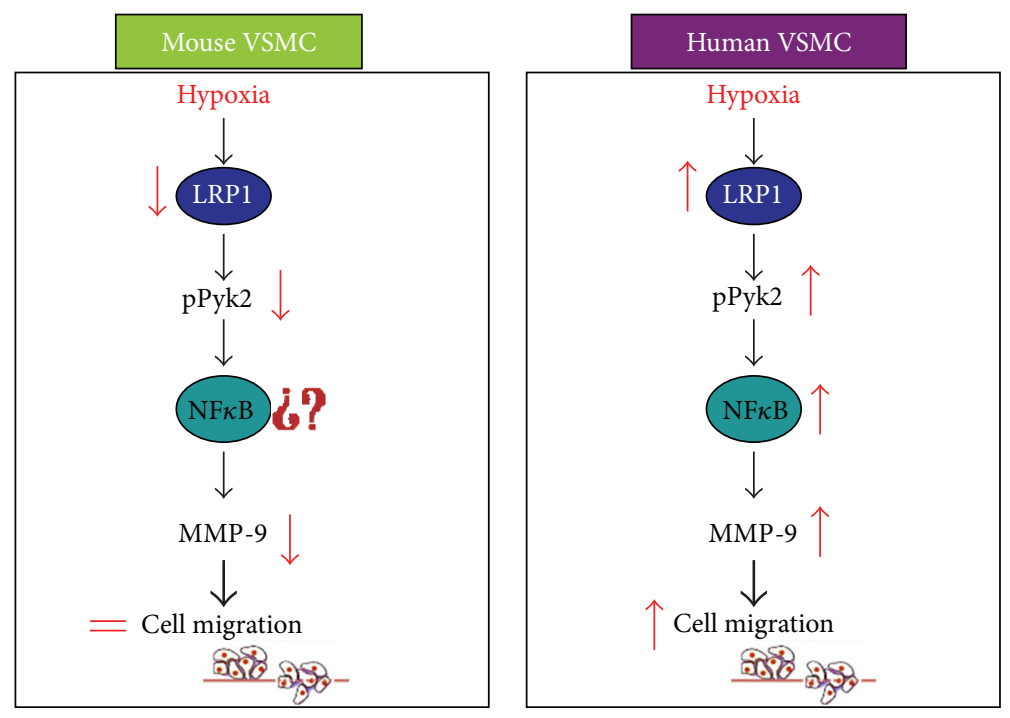

FIGURE 5: Representative scheme comparing hypoxia-induced alterations in mouse and human VSMC.

cytoplasmic levels while LRP1 downregulation in mVSMC will facilitate decreased calcium intracellular levels. In the present study, we also showed that the inverse effect of LRP1/pPyk2 axis is associated with a differential effect of hypoxia on MMP-9 expression and activation. It is clear that, during hypoxia, MMP-9 activation or inactivation is species-type specific and, apparently, depends, respectively, on the upregulatory or downregulatory effects of hypoxia on LRP1-pPyk2 levels. Our results contribute to explaining the controversial findings in the literature concerning the effect of hypoxia on MMP-9 levels. MMP-9 production is downregulated by hypoxia in human monocyte-derived dendritic cells [30] but upregulated in both tumoral [31] and cardiac muscle cells [32].

Hypoxia-induced MMP-9 activation was concomitant with an increased hVSMC migratory capacity. Surprisingly, mVSMC migrated under hypoxic conditions despite the hypoxic downregulatory effect on MMP-9 activation. The migratory capacity of hypoxic mVSMC was strongly reduced by inhibition of ERK1,2 phosphorylation, suggesting that ERK1,2 phosphorylation may modulate mVSMC migration in a MMP-9 independent manner. However, the time-course of ERK1,2 phosphorylation like that of MMP9 activation differs in normoxic and hypoxic mVSMC, indicating that the modulation of this pathway strongly depends on $\mathrm{O}_{2}$ availability in mVSMC.

In summary, our results show a crucial impact of the LRP1-pPyk2-MMP-9 axis on vascular cell migration (summarized in Figure 5) and highlight that the direct extrapolation from murine models to humans is not suitable at least for mechanisms underlying hypoxia-related vascular diseases.

\section{Conflict of Interests}

The authors declare that there is no conflict of interests regarding the publication of this paper.

\section{Acknowledgments}

The authors thank the Heart Transplant Team at the Cardiology and Cardiac Surgery Service at Hospital de la Santa Creu i Sant Pau. This work was funded by FIS PI11/00747 from Instituto de Salud Carlos III and cofinanced by the European Fund for Regional Development and by Red Investigación Cardiovascular (RD RD12/0042/0027). E. Revuelta-López is financed by AGAUR from Generalitat de Catalunya (DGR 2013 FI_B 01008) and D. de Gonzalo-Calvo is financed by Red Investigación Cardiovascular (RD RD12/0042/0027).

\section{References}

[1] P. Libby and E. Folco, "Tension in the plaque: hypoxia modulates metabolism in atheroma," Circulation Research, vol. 109, no. 10, pp. 1100-1102, 2011.

[2] A. J. Lusis, "Atherosclerosis," Nature, vol. 407, no. 6801, pp. 233241, 2000.

[3] V. Llorente-Cortés, M. Otero-Viñas, S. Camino-López, P. Costales, and L. Badimon, "Cholesteryl esters of aggregated LDL are internalized by selective uptake in human vascular smooth muscle cells," Arteriosclerosis, Thrombosis, and Vascular Biology, vol. 26, no. 1, pp. 117-123, 2006.

[4] M. Otero-Viñas, V. Llorente-Cortés, E. Peña, T. Padró, and L. Badimon, "Aggregated low density lipoproteins decrease metalloproteinase- 9 expression and activity in human coronary smooth muscle cells," Atherosclerosis, vol. 194, no. 2, pp. 326333, 2007

[5] A. C. Newby, "Matrix metalloproteinases regulate migration, proliferation, and death of vascular smooth muscle cells by degrading matrix and non-matrix substrates," Cardiovascular Research, vol. 69, no. 3, pp. 614-624, 2006.

[6] E. Hahn-Dantona, J. F. Ruiz, P. Bornstein, and D. K. Strickland, "The low density lipoprotein receptor-related protein modulates levels of matrix metalloproteinase 9 (MMP-9) by mediating its cellular catabolism," Journal of Biological Chemistry, vol. 276, no. 18, pp. 15498-15503, 2001. 
[7] V. Montel, A. Gaultier, R. D. Lester, W. M. Campana, and S. L. Gonias, "The low-density lipoprotein receptor-related protein regulates cancer cell survival and metastasis development," Cancer Research, vol. 67, no. 20, pp. 9817-9824, 2007.

[8] C. D. Overton, P. G. Yancey, A. S. Major, M. F. Linton, and S. Fazio, "Deletion of macrophage LDL receptor-related protein increases atherogenesis in the mouse," Circulation Research, vol. 100, no. 5, pp. 670-677, 2007.

[9] E. Mantuano, G. Inoue, X. Li et al., "The hemopexin domain of matrix metalloproteinase- 9 activates cell signaling and promotes migration of schwann cells by binding to low-density lipoprotein receptor-related protein," Journal of Neuroscience, vol. 28, no. 45, pp. 11571-11582, 2008.

[10] H. O. Jung, J. S. Uhm, S. M. Seo et al., "Angiotensin II-induced smooth muscle cell migration is mediated by LDL receptorrelated protein 1 via regulation of matrix metalloproteinase 2 expression," Biochemical and Biophysical Research Communications, vol. 402, no. 4, pp. 577-582, 2010.

[11] J. Castellano, R. Aledo, J. Sendra et al., "Hypoxia stimulates low-density lipoprotein receptor-related protein-1 expression through hypoxia-inducible factor- $1 \alpha$ in human vascular smooth muscle cells," Arteriosclerosis, Thrombosis, and Vascular Biology, vol. 31, no. 6, pp. 1411-1420, 2011.

[12] T. Nassar, S. Akkawi, R. Bar-Shavit et al., "Human $\alpha$-defensin regulates smooth muscle cell contraction: a role for lowdensity lipoprotein receptor-related protein $/ \alpha_{2}$-macroglobulin receptor," Blood, vol. 100, no. 12, pp. 4026-4032, 2002.

[13] D. Beitner-Johnson, T. Ferguson, R. T. Rust, S. Kobayashi, and D. E. Millhorn, "Calcium-dependent activation of Pyk2 by hypoxia," Cellular Signalling, vol. 14, no. 2, pp. 133-137, 2002.

[14] E. Revuelta-López, J. Castellano, S. Roura et al., "Hypoxia induces metalloproteinase-9 activation and human vascular smooth muscle cell migration through low-density lipoprotein receptor-related protein 1-mediated Pyk2 phosphorylation," Arteriosclerosis, Thrombosis, and Vascular Biology, vol. 33, no. 12, pp. 2877-2887, 2013.

[15] Y. Nakashima, H. Fujii, S. Sumiyoshi, T. N. Wight, and K. Sueishi, "Early human atherosclerosis: accumulation of lipid and proteoglycans in intimal thickenings followed by macrophage infiltration," Arteriosclerosis, Thrombosis, and Vascular Biology, vol. 27, no. 5, pp. 1159-1165, 2007.

[16] S. Allahverdian, P. S. Pannu, and G. A. Francis, "Contribution of monocyte-derived macrophages and smooth muscle cells to arterial foam cell formation," Cardiovascular Research, vol. 95, no. 2, pp. 165-172, 2012.

[17] V. Llorente-Cortés, J. Martínez-González, and L. Badimon, "LDL receptor-related protein mediates uptake of aggregated LDL in human vascular smooth muscle cells," Arteriosclerosis, Thrombosis, and Vascular Biology, vol. 20, no. 6, pp. 1572-1579, 2000.

[18] V. Llorente-Cortés, M. Otero-Viñas, S. Sánchez, C. Rodríguez, and L. Badimon, "Low-density lipoprotein upregulates lowdensity lipoprotein receptor-related protein expression in vascular smooth muscle cells: Possible involvement of sterol regulatory element binding protein-2-dependent mechanism," Circulation, vol. 106, no. 24, pp. 3104-3110, 2002.

[19] V. Andrés, J. Ureña, E. Poch, D. Chen, and D. Goukassian, "Role of Sp1 in the induction of p27 gene expression in vascular smooth muscle cells in vitro and after balloon angioplasty," Arteriosclerosis, Thrombosis, and Vascular Biology, vol. 21, no. 3, pp. 342-347, 2001.
[20] J. F. Bentzon and E. Falk, "Atherosclerotic lesions in mouse and man: is it the same disease?" Current Opinion in Lipidology, vol. 21, no. 5, pp. 434-440, 2010.

[21] R. Cal, J. Castellano, E. Revuelta-López et al., "Low-density lipoprotein receptor-related protein 1 mediates hypoxiainduced very low density lipoprotein-cholesteryl ester uptake and accumulation in cardiomyocytes," Cardiovascular Research, vol. 94, no. 3, pp. 469-479, 2012.

[22] Y. V. Liu, M. E. Hubbi, F. Pan et al., "Calcineurin promotes hypoxia-inducible factor $1 \alpha$ expression by dephosphorylating RACK1 and blocking RACK1 dimerization," Journal of Biological Chemistry, vol. 282, no. 51, pp. 37064-37073, 2007.

[23] M. X. Zhu, J. Ma, J. Parrington, P. J. Calcraft, A. Galione, and A. M. Evans, "Calcium signaling via two-pore channels: local or global, that is the question," The American Journal of Physiology: Cell Physiology, vol. 298, no. 3, pp. C430-C441, 2010.

[24] M. D. Schaller, "Cellular functions of FAK kinases: insight into molecular mechanisms and novel functions," Journal of Cell Science, vol. 123, no. 7, pp. 1007-1013, 2010.

[25] P. Costales, R. Aledo, S. Vérnia et al., "Selective role of sterol regulatory element binding protein isoforms in aggregated LDL-induced vascular low density lipoprotein receptor-related protein-1 expression," Atherosclerosis, vol. 213, no. 2, pp. 458$468,2010$.

[26] G. Karakiulakis, E. Papakonstantinou, A. J. Aletras, M. Tamm, and M. Roth, "Cell type-specific effect of hypoxia and plateletderived growth factor-BB on extracellular matrix turnover and its consequences for lung remodeling," The Journal of Biological Chemistry, vol. 282, no. 2, pp. 908-915, 2007.

[27] B. D. Kelly, S. F. Hackett, K. Hirota et al., "Cell type-specific regulation of angiogenic growth factor gene expression and induction of angiogenesis in nonischemic tissue by a constitutively active form of hypoxia-inducible factor 1," Circulation Research, vol. 93, no. 11, pp. 1074-1081, 2003.

[28] R. D. Bell, R. Deane, N. Chow et al., "SRF and myocardin regulate LRP-mediated amyloid- $\beta$ clearance in brain vascular cells," Nature Cell Biology, vol. 11, no. 2, pp. 143-153, 2009.

[29] O. M. Andersen, H. Vorum, B. Honoré, and H. C. Thøgersen, " $\mathrm{Ca}^{2+}$ binding to complement-type repeat domains 5 and 6 from the low-density lipoprotein receptor-related protein," BMC Biochemistry, vol. 4, article 7, 2003.

[30] W. Zhao, S. Darmanin, Q. Fu et al., "Hypoxia suppresses the production of matrix metalloproteinases and the migration of human monocyte-derived dendritic cells," European Journal of Immunology, vol. 35, no. 12, pp. 3468-3477, 2005.

[31] J. Y. Choi, Y. S. Jang, S. Y. Min, and J. Y. Song, “Overexpression of MMP-9 and hif-lalpha in breast cancer cells under hypoxic conditions," Journal of Breast Cancer, vol. 14, no. 2, pp. 88-95, 2011.

[32] A. M. Romanic, C. L. Burns-Kurtis, B. Gout, I. BerrebiBertrand, and E. H. Ohlstein, "Matrix metalloproteinase expression in cardiac myocytes following myocardial infarction in the rabbit," Life Sciences, vol. 68, no. 7, pp. 799-814, 2001. 


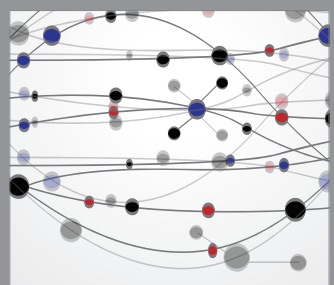

The Scientific World Journal
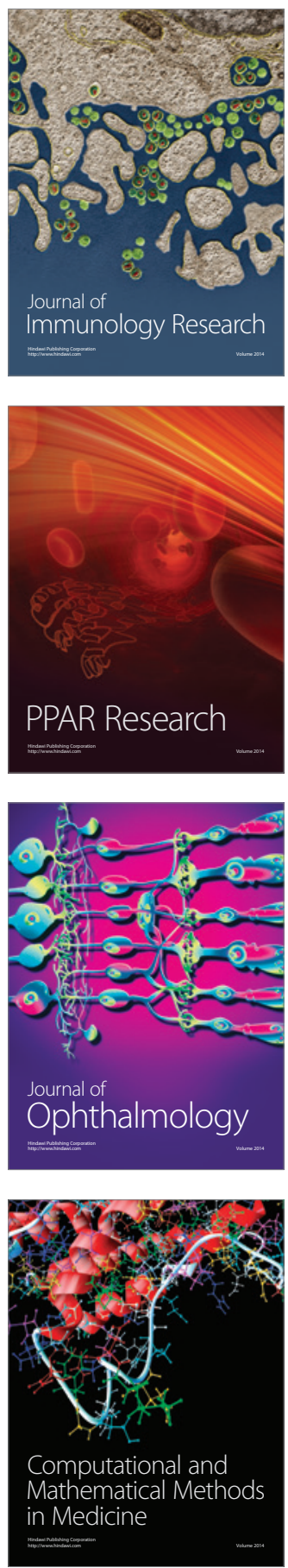

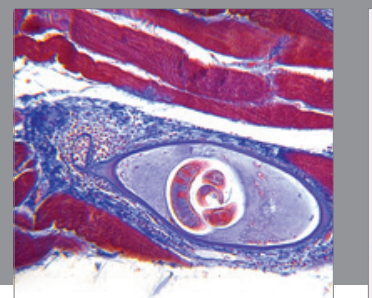

Gastroenterology

Research and Practice
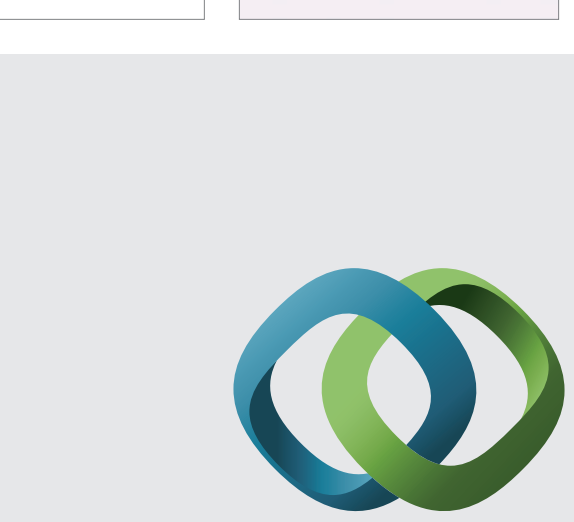

\section{Hindawi}

Submit your manuscripts at

http://www.hindawi.com
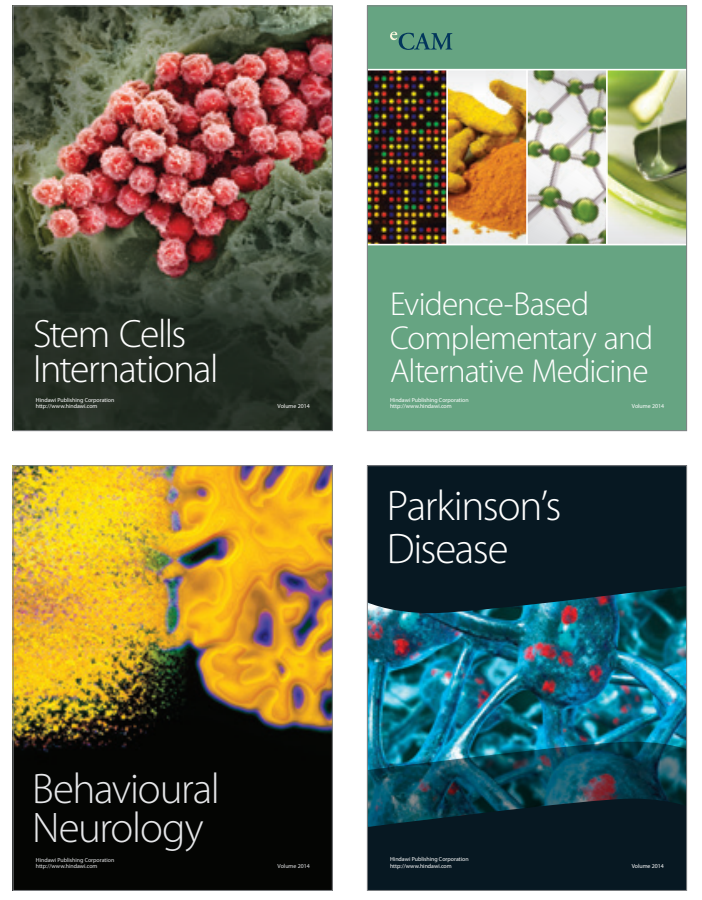
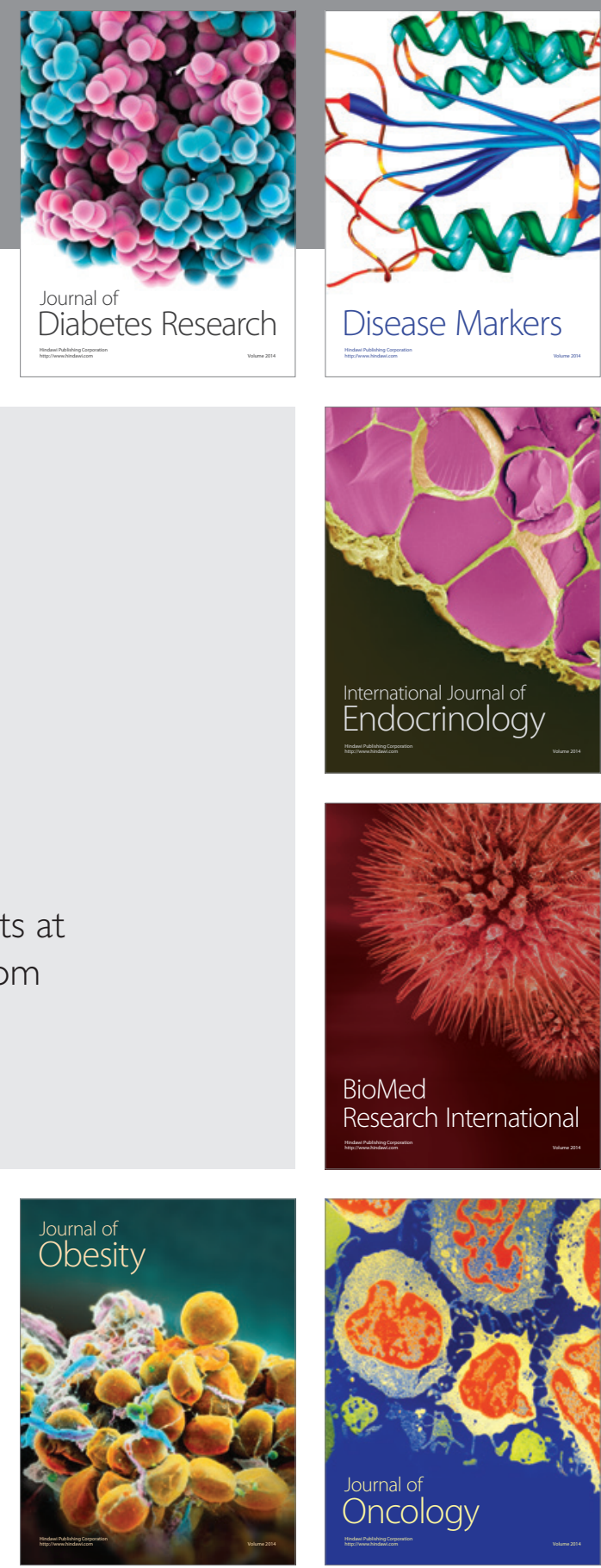

Disease Markers
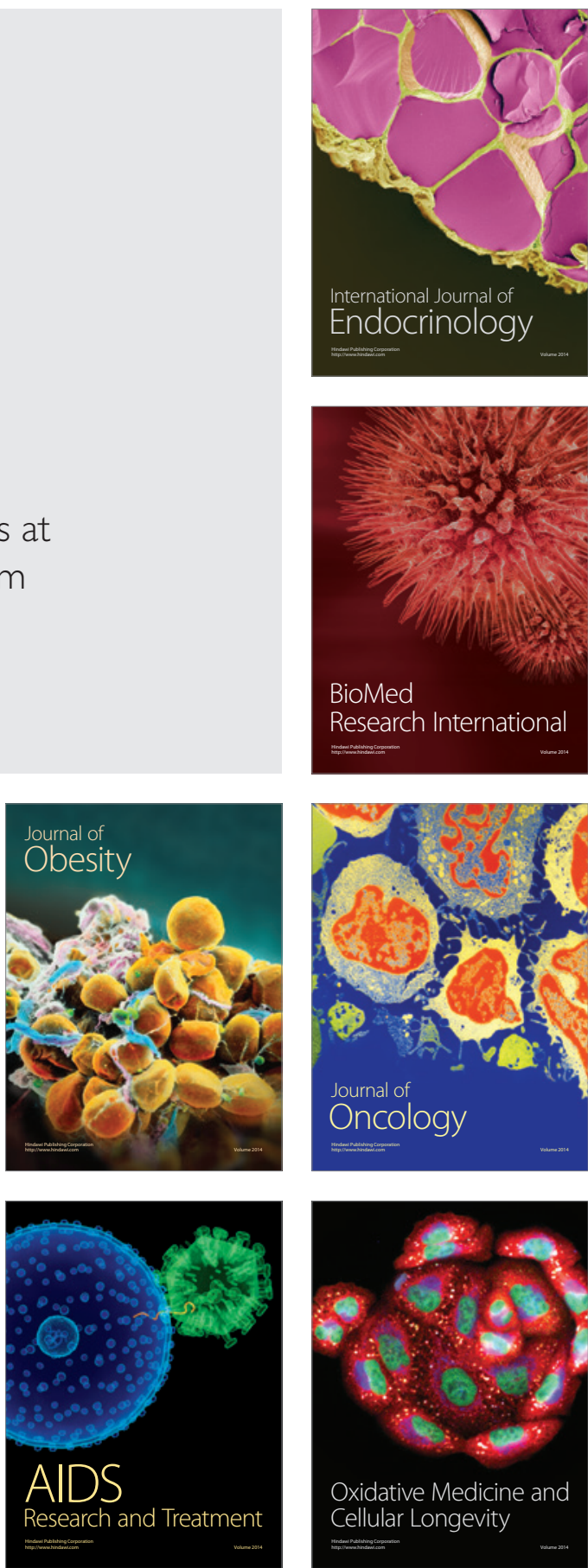